\title{
Ischemic Lesion Water Uptake in Acute Stroke: Is Blood Glucose Related to Cause and Effect?
}

\author{
Gabriel Broocks, ${ }^{\mathrm{a}}$ Andre Kemmling, ${ }^{\mathrm{b}, \mathrm{c}}$ Jens Aberle, ${ }^{\mathrm{d}}$ Helge Kniep, ${ }^{\mathrm{a}}$ Matthias Bechstein, ${ }^{\mathrm{a}}$ \\ Fabian Flottmann, ${ }^{\mathrm{a}}$ Hannes Leischner, ${ }^{\mathrm{a}}$ Tobias D. Faizy, ${ }^{\mathrm{a}}$ Jawed Nawabi, ${ }^{\mathrm{a}}$ Gerhard Schön, ${ }^{\mathrm{e}}$ Peter Sporns, ${ }^{\mathrm{f}}$ \\ Götz Thomalla, ${ }^{\mathrm{g}}$ Jens Fiehler, ${ }^{\mathrm{a}}$ Uta Hanning ${ }^{\mathrm{a}}$ \\ a'Department of Diagnostic and Interventional Neuroradiology, University Medical Center Hamburg-Eppendorf, Hamburg, Germany \\ ${ }^{b}$ Department of Neuroradiology, Westpfalz-Klinikum, Kaiserslautern, Germany \\ 'Faculty of Medicine Mannheim, University of Heidelberg, Mannheim, Germany \\ ${ }^{d}$ Department of Endocrinology and Diabetology, University Medical Center Hamburg-Eppendorf, Hamburg, Germany \\ eInstitute of Medical Biometry and Epidemiology, University Medical Center Hamburg-Eppendorf, Hamburg, Germany \\ fDepartment of Radiology, University Hospital Muenster, Muenster, Germany \\ ${ }^{9}$ Department of Neurology, University Medical Center Hamburg-Eppendorf, Hamburg, Germany
}

\section{Dear Sir:}

Functional outcome after thrombectomy in patients with acute ischemic stroke still varies significantly. Higher blood glucose levels (BGL) have been associated with poor clinical outcome, but the pathophysiological causes are not yet understood. Prior animal studies suggested that hyperglycemia accelerates the transformation of ischemic penumbra into infarction resulting in larger infarcts. ${ }^{1}$ Moreover, altered blood-brain barrier permeability or increased lactic acid production in the ischemic tissue have been discussed as potential pathophysiological causes. ${ }^{2}$ Recently, higher blood glucose has been described as an important predictor for early cerebral brain edema; however, in the referred study, edema was only determined using a visual three-step approach, which primarily assessed infarct size. ${ }^{3}$ Nonetheless, this study introduced early brain edema as a possible factor resulting from elevated BGL, whose pathophysiology is not yet entirely understood.

The purpose of this pilot study was to investigate the relationship of admission blood glucose and early cerebral edema in stroke patients using a quantitative computed tomography (CT) based method to determine ischemic lesion water uptake. ${ }^{4}$

We retrospectively screened all ischemic stroke patients with large vessel occlusion of the middle cerebral artery territory admitted between February 2015 and March 2018 at the local high-volume tertiary stroke center. The a priori defined inclusion criteria can be found in the Supplementary Material. Baseline clinical characteristics and demographic information were extracted from the medical records, including time from onset to admission and blood glucose at admission, as well as anti-diabetic medication including insulin. A history of diabetes mellitus was retrieved from clinical documentation. Functional outcome was extracted from the registry using modified Rankin Scale (mRS) scores after 90 days. We used a standardized procedure to quantify early net lesion water uptake (NWU) in the ischemic core lesion at the time of admission imaging as reported elsewhere. ${ }^{5-7}$ The relationship of BGL and early edema (e.g., NWU in admission $\mathrm{CT}$ ) was analyzed using multivariable linear regression adjusted for Alberta Stroke Program Early CT Score (ASPECTS), age, and National Institute of Health Stroke Scale (NIHSS). The association of BGL on clinical outcome was analyzed using univariable and multivariable logistic regression analysis with backward selection adjusted for age, NIHSS, ASPECTS, NWU, and recanalization status. Details on statistical analyses can be found in the Supplementary Material.

In this pilot study, 165 patients fulfilled all inclusion criteria and were analyzed (Table 1). The median age was 76 (interquartile range [IQR], 65 to 82 ) and 69 patients (42\%) were women. In 30 patients, a known history of diabetes mellitus type 2 was found in medical documentation (18\%). In these patients, the mean BGL at admission was significantly higher $(157 \mathrm{mg} / \mathrm{dL}$ vs. $127 \mathrm{mg} / \mathrm{dL}, P<0.0001)$. Regarding existing diabetes medication, eight patients had subcutaneous insulin in their medication list, 
Table 1. Patient characteristics

\begin{tabular}{lccc}
\hline Characteristic & $\begin{array}{c}\text { Modified } \\
\text { Rankin Scale } \\
0-2\end{array}$ & $\begin{array}{c}\text { Modified } \\
\text { Rankin Scale }\end{array}$ & $P-6$ \\
\hline Subjects & $61(36.4)$ & $105(63.6)$ & \\
Admission NIHSS & $14(11-18)$ & $18(15-20)$ & $<0.01$ \\
ASPECTS & $8(7-9)$ & $7(5-8)$ & $<0.01$ \\
Administered intravenous lysis & $49(81)$ & $55(52)$ & $<0.01$ \\
Follow-up infarct volume (mL) & $12.4 \pm 28.5$ & $38.5 \pm 83.1$ & 0.07 \\
TICl score 2b/3 & $60(98)$ & $88(84)$ & 0.02 \\
Net water uptake in admission & $6.5 \pm 4.7$ & $9.7 \pm 5.1$ & $<0.01$ \\
$\quad$ CT (\%) & & & \\
Blood glucose level at admis- & $116.4 \pm 24.1$ & $137.2 \pm 36.6$ & $<0.01$ \\
$\quad$ sion (mg/dL) & & & \\
History of diabetes mellitus & $8(13)$ & $19(18)$ & 0.61 \\
Insulin medication & $1(1.6)$ & $6(5.7)$ & 0.26 \\
Antidiabetic drugs & $1(1.6)$ & $7(6.6)$ & 0.37 \\
\hline
\end{tabular}

Values are presented as number (\%), median (interquartile range), or mean \pm standard deviation.

NIHSS, National Institute of Health Stroke Scale; ASPECTS, Alberta Stroke Program Early CT Score; $\mathrm{TICl}$, thrombolysis in cerebral infarctions; CT, computed tomography.

five patients with metformin, four patients with gliptins, and one patient with sulfonylurea. The mean \pm standard deviation (SD) time from symptom onset to admission imaging was $3.1 \pm 1.8$ hours and the median NIHSS was 16 (IOR, 13 to 19 ). The median ASPECTS was 8 (IQR, 6 to 9). One hundred and thirty-one patients (79\%) received successful endovascular recanalization with $\mathrm{TICl} 2 \mathrm{~b} / 3$. Patients with a history of diabetes had no significant difference in early formation of ischemic edema than patients without (mean NWU $10.6 \%$ vs. $9.8 \%$, $P=0.44)$. When using BGL of $140 \mathrm{mg} / \mathrm{dL}$ as cut-off to distinguish two patient groups, however, we found significant differences when comparing these groups. The mean \pm SD NWU for patients with $B G L<140 \mathrm{mg} / \mathrm{dL}$ was significantly lower than NWU in patients with $B G L>140 \mathrm{mg} / \mathrm{dL}$ (112.3 mg/dL [15.2] vs. $171.1 \mathrm{mg} / \mathrm{dL}$ [33.8], $P<0.0001)$. In multivariable linear regression analysis, the association of BGL on NWU was assessed adjusted for ASPECTS, NIHSS, and age. For every $10 \mathrm{mg} / \mathrm{dL}$ increase in BGL, NWU significantly increased by $0.56 \%(95 \%$ confidence interval $[\mathrm{Cl}, 0.33$ to $0.79 ; P<0.001$ ) (Figure 1).

Univariate and multivariable logistic regression analysis was performed to predict good outcome (mRS 0 to 2) using BGL, NIHSS, age, NWU, recanalization status and ASPECTS as independent variables. Higher BGL significantly reduced the likelihood of good outcome of $3 \%$ per $1 \mathrm{mg} / \mathrm{dL}$ increase (odds ratio, $0.97 ; 95 \% \mathrm{Cl}, 0.96$ to $0.99 ; P=0.0006$ ) (Figure 2).

The aim of this pilot study was to investigate the relationship

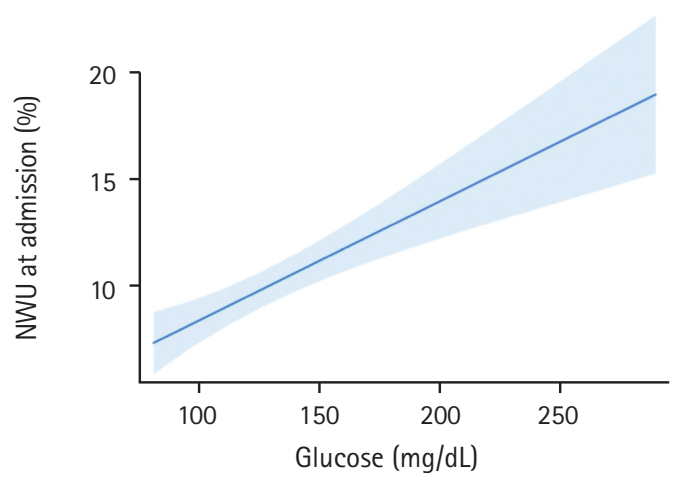

Figure 1. Association of blood glucose with early net lesion water uptake (NWU). Association of blood glucose (x axis) with ischemic NWU (y axis) in the admission computed tomography. Shaded area indicates a 95\% confidence interval.

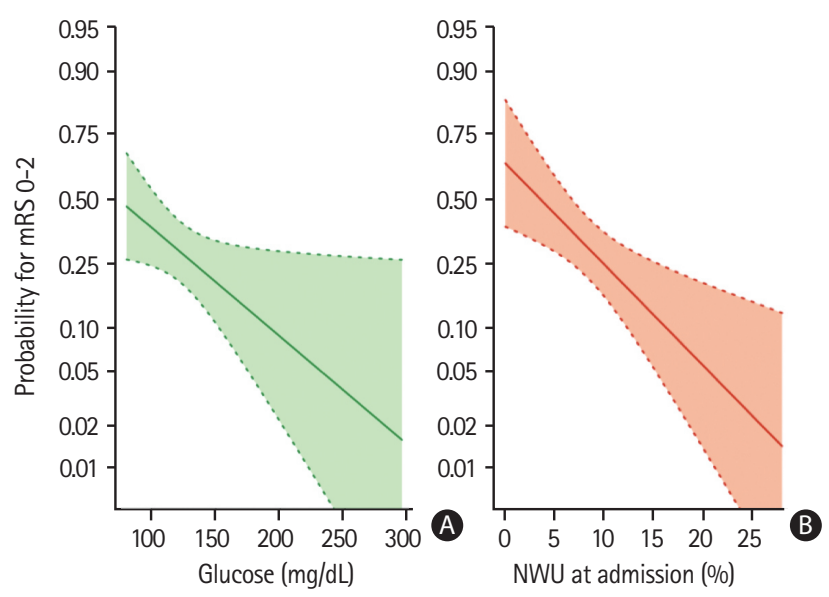

Figure 2. Multivariable logistic regression analysis to display (A) the impact of admission blood glucose ( $\mathrm{mg} / \mathrm{dL}$ ) (B) and early net lesion water uptake (NWU) at admission (\%) on clinical outcome (probability for functional independence), adjusted for age, National Institute of Health Stroke Scale, and Alberta Stroke Program Early CT Score. Shaded area indicates a 95\% confidence interval. mRS, modified Rankin Scale.

of admission blood glucose, early ischemic brain edema and clinical outcome in ischemic stroke patients. The main findings of this exploratory analysis was that increasing BGL was significantly associated with higher early brain edema and worse functional outcome assessed by mRS after 90 days. To our knowledge, the present pilot study is the first that investigated the relationship of BGL and early ischemic edema using a quantitative imaging biomarker. ${ }^{5} \mathrm{~A}$ lately published paper observed that elevated BGL was an important predictor for early ischemic edema, but these findings were only based on visual rating primarily describing lesion size. ${ }^{3}$ Our pilot study confirmed prior observations that higher levels of blood glucose were associated with reduced functional independence, adjusted for baseline clinical parameter and treatment. Early cerebral brain edema might be the link 
between elevated BGL and poor outcome. In the future, quantification of early ischemic edema might be used to monitor treatment effects of adjuvant anti-edematous treatment, such as glyburide. ${ }^{8}$ It is important to realize that applying antidiabetic drugs or insulin in clinical trials did not improve functional outcome although animal studies often showed promising results. ${ }^{9}$ Likewise, the use of further neuroprotectants for adjuvant stroke treatment often failed. In contrast to clinical trials, $>80 \%$ of prior preclinical studies examined the neuroprotectant in animal models in combination with complete vessel reperfusion. Considering the low recanalization rates in patients with large vessel occlusion and intravenous lysis, the disappointing results of several clinical trials testing adjuvant treatment strategies may be recognizable. Therefore, future clinical trials could combine vessel reperfusion with neuroprotectants or anti-diabetic treatment in patients with hyperglycemia.

Limitations of this pilot study include the small number of patients due to strict inclusion and exclusion criteria. Furthermore, hyperglycemia is a dynamic process, and an isolated blood glucose value may not be sufficient to capture the whole complexity of ischemic brain. Finally, future studies should especially investigative the relationship between ischemic brain edema, BGL and collateral status as potential important mediator, as recently described..$^{10}$

In conclusion, this study confirms the association between higher levels of blood glucose and reduced likelihood of functional independence, adjusted for baseline clinical parameters and treatment. Early cerebral brain edema might be a link between elevated BGL and poor outcome.

\section{Supplementary materials}

Supplementary materials related to this article can be found online at https://doi.org/10.5853/jos.2019.01935.

\section{References}

1. Martini SR, Kent TA. Hyperglycemia in acute ischemic stroke: a vascular perspective. J Cereb Blood Flow Metab 2007;27:435451.

2. Lu G, Ren ZO, Zhang JX, Zu Q0, Shi HB. Effects of diabetes mellitus and admission glucose in patients receiving mechanical thrombectomy: a systematic review and metaanalysis. Neurocrit Care 2018;29:426-434.

3. Thorén $M$, Azevedo E, Dawson J, Egido JA, Falcou A, Ford GA, et al. Predictors for cerebral edema in acute ischemic stroke treated with intravenous thrombolysis. Stroke 2017;48:2464-2471.
4. Broocks G, Flottmann F, Scheibel A, Aigner A, Faizy TD, Hanning $U$, et al. Quantitative lesion water uptake in acute stroke computed tomography is a predictor of malignant infarction. Stroke 2018;49:1906-1912.

5. Broocks G, Flottmann F, Ernst M, Faizy TD, Minnerup J, Siemonsen $\mathrm{S}$, et al. Computed tomography-based imaging of voxel-wise lesion water uptake in ischemic brain: relationship between density and direct volumetry. Invest Radiol 2018;53:207-213.

6. Broocks G, Hanning U, Flottmann F, Schönfeld M, Faizy TD, Sporns $P$, et al. Clinical benefit of thrombectomy in stroke patients with low ASPECTS is mediated by oedema reduction. Brain 2019;142:1399-1407.

7. Minnerup J, Broocks G, Kalkoffen J, Langner S, Knauth $M$, Psychogios MN, et al. Computed tomography-based quantification of lesion water uptake identifies patients within 4.5 hours of stroke onset: a multicenter observational study. Ann Neurol 2016;80:924-934.

8. Sheth KN, Elm JJ, Molyneaux BJ, Hinson H, Beslow LA, Sze GK, et al. Safety and efficacy of intravenous glyburide on brain swelling after large hemispheric infarction (GAMES$\mathrm{RP}$ ): a randomised, double-blind, placebo-controlled phase 2 trial. Lancet Neurol 2016;15:1160-1169.

9. Johnston KC, Bruno A, Pauls Q, Hall CE, Barrett KM, Barsan W, et al. Intensive vs standard treatment of hyperglycemia and functional outcome in patients with acute ischemic stroke: the SHINE randomized clinical trial. JAMA 2019;322:326-335.

10. Kim JT, Liebeskind DS, Jahan R, Menon BK, Goyal M, Nogueira RG, et al. Impact of hyperglycemia according to the collateral status on outcomes in mechanical thrombectomy. Stroke 2018;49:2706-2714.

Correspondence: Gabriel Broocks

Department of Diagnostic and Interventional Neuroradiology, University Medical Center Hamburg-Eppendorf, Martinistrasse 52, Hamburg 20246, Germany

Tel: +49-152228-35101

Fax: +49-407410-40114

E-mail: g.broocks@uke.de

Received: July 6, 2019

Revised: August 7, 2019

Accepted: August 12, 2019

Jens Fiehler: Research support: German Ministry of Science and Education (BMBF and BMWi), German Research Foundation (DFG), European Union (EU), Hamburgische Investitions- und Förderbank (IFB), Medtronic, Microvention, Philips, Stryker, Consultant for: Acandis, Boehringer Ingelheim, Cerenovus, Covidien, Evasc Neurovascular, MD Clinicals, Medtronic, Medina, Microvention, Penumbra, Route92, Stryker, Transverse Medical. Götz Thomalla: Consultant for Acandis, Bayer Healthcare, Boehringer Ingelheim, BristolMyersSquibb/Pfizer, Covidien, Glaxo Smith Kline; lead investigator of the WAKE-UP study; Principal Investigator of the THRILL study; Grants by the European Union (Grant No. 278276 und 634809) and Deutsche Forschungsgemeinschaft (SFB 936, Projekt C2). 


\section{Supplementary Material}

\section{Patient selection}

The a priori defined inclusion criteria were: (1) acute ischemic stroke with large vessel occlusion of the distal internal carotid artery or middle cerebral artery confirmed by multimodal computed tomography (CT) on admission (non-enhanced CT [NECT], CT angiography [CTA], and CT perfusions [CTP]) within 6 hours from symptom onset; (2) visually evident early infarct lesion as indicated by ischemic hypoattenuation in admission NECT and/or perfusion lesion with reduced cerebral blood volume in CTP; (3) subsequently performed endovascular procedure with documented Thrombolysis In Cerebral Infarctions (TICI) score; (4) documented time from symptom onset to imaging and National Institute of Health Stroke Scale (NIHSS) score; and (5) absence of intracranial hemorrhage and preexisting infarctions in admission NECT. Baseline clinical characteristics and demographic information were extracted from the medical records, including time from onset to admission and blood glucose at admission, as well as anti-diabetic medication including insulin. A history of diabetes mellitus was retrieved from clinical documentation. Functional outcome was extracted from the registry using modified Rankin Scale (mRS) scores after 90 days.

\section{Imaging}

Patients received a comprehensive stroke imaging protocol by multimodal CT at admission with NECT and CTA and additional CTP in equal order on an iCT 25 scanner (Philips Healthcare, Best, The Netherlands); NECT: collimation $64 \times 0.625$, pitch 0.297 , rotation time 0.4 second, field of view (FOV) $270 \mathrm{~mm}$, tube voltage $120 \mathrm{kV}$, tube current $300 \mathrm{~mA}, 4.0 \mathrm{~mm}$ slice reconstruction; CTA: collimation $64 \times 0.625$, pitch 0.985 , rotation time 0.4 second, FOV $220 \mathrm{~mm}$, tube voltage $120 \mathrm{kV}, 300 \mathrm{mAs}$, $2.0 \mathrm{~mm}$ slice reconstruction, $5 \mathrm{~mm}$ maximum intensity projec- tion reconstruction with $1 \mathrm{~mm}$ increment; CTP: collimation $64 \times 1.25$, rotation time 0.5 second, FOV $220 \mathrm{~mm}$, tube current $80 \mathrm{kV}$, tube current $140 \mathrm{mAs}, 5 \mathrm{~mm}$ slice reconstruction, slice sampling rate 1.8 seconds, scan time 72 seconds, biphasic injection with $40 \mathrm{~mL}$ of highly iodinated contrast medium with $400 \mathrm{mM} / \mathrm{mL}$ injected with $6 \mathrm{~mL} / \mathrm{sec}$ followed by $40 \mathrm{~mL} \mathrm{NaCl}$ chaser bolus. Perfusion datasets were inspected for quality and excluded in case of severe motion artefacts.

\section{Statistical analysis}

Continuous variables are presented as means, confidence intervals of means, standard deviations or medians and ranges. Kolmogorov-smirnov tests were used to determine if the data sets were well-modeled by a normal distribution. Patients with good ( $m R S 0$ to 2) outcome were compared to patients with $m R S>2$ in Table 1 using Student t-tests (normal distribution) or MannWhitney $U$ tests (non-normal distribution). Moreover, patients below or above $140 \mathrm{mg} / \mathrm{dL}$ BGL were compared. The relationship of BGL on early edema (e.g., NWU in admission CT) was analyzed using multivariable linear regression adjusted for Alberta Stroke Program Early CT Score (ASPECTS), age, and NIHSS.

The association of BGL on clinical outcome was analyzed using univariable and multivariable logistic regression analysis with backward selection adjusted for collateral score, age, NIHSS, ASPECTS, NWU, and recanalization status. The dependent variable was functional independence (mRS 0 to 2).

A statistcally significant difference was accepted at a $P$-value of less than 0.05. Analyses were performed using MedCalc version 11.5.1.0 (MedCalc statistical software, Mariakerke, Belgium) and R (R Core Team, R: A Language and Environment for Statistical Computing; R Foundation for Statistical Computing, Vienna, Austria; 2017).

\section{Data availability statement}

The data that support the findings of this study are available from the corresponding author upon reasonable request. 